\title{
LA INFANCIA EN EL PALEOLÍTICO SUPERIOR: PRESENCIA Y REPRESENTACIÓN
}

\author{
Chilhood in the Upper Paleolithic: Presence and Representation \\ Alberto Lombo Montañés (albertrisa13@hotmail.com) ${ }^{1}$ \\ Clara Hernando Álvarez (clara85@usal.es) ${ }^{2}$ \\ Leyre Alconchel Navarro (leyrealconchel@gmail.com) ${ }^{1}$ \\ Paloma Lanau Hernáez (palomalanau@gmail.com) ${ }^{1}$
}

Fecha de recepción: $14-\mathrm{IV}-2013$

Fecha de aceptación: 30-IV-2013

\begin{abstract}
RESUMEN: En los últimos años se han multiplicado los estudios sobre la infancia en el pasado, las huellas dejadas en el registro material por los individuos infantiles y su papel en la comunidad. Este trabajo pretende llamar la atención sobre las múltiples representaciones de la infancia en el registro arqueológico del Paleolítico Superior. Con este objetivo se analizan las que se han definido en la bibliografía especializada como representaciones de individuos infantiles en el arte mueble y parietal, los enterramientos o las huellas de pies y manos consecuencia del tránsito de los niños por las cuevas. En el apartado metodológico se hace una aproximación crítica a las características que permiten adscribir estas manifestaciones al mundo infantil y se discute sobre las posibles inferencias que se pueden realizar acerca de la infancia a partir de los datos disponibles.
\end{abstract}

Palabras clave: Infancia, Arqueología, Paleolítico superior, Europa.

АвSTRACT: In recent years the studies relating to the childhood in the past, the traces of children activity in the material culture and their role in the community have been increased. This work draws attention to the multiple representations of the childhood through the

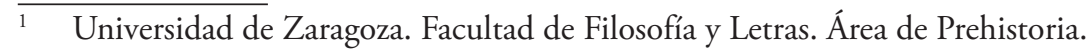

2 Universidad de Salamanca. Departamento de Prehistoria, Historia Antigua y Arqueología. 
Upper Paleolithic archaeological remains. With this aim, we analyze some of the evidences attached to childhood in the scientific bibliography, such as children figures in the rock and mobiliary art, burials and footprints left in caves. The methodological section includes a critical approach to the features which may allow torecognize the child representations and also a discussion of the possibilities for research from the available information.

Keywords: Childhood, Archaeology, Upper Paleolithic, Europe.

\section{INTRODUCCIÓN}

En los últimos tiempos, uno de los objetos (sujetos) principales de investigación arqueológica han sido los individuos infantiles como productores de registro arqueológico o como participantes activos de éste (POLITIS, 1998; SACCHI, 2010). La arqueología postprocesual ha posibilitado la «visibilización» de las mujeres a través de estudios de género ${ }^{3}$ en primer lugar; para, a continuación, acercarse al silenciado mundo de la infancia (LILLEHAMMER, 2010). Así, en la década de los 70, comienzan los interrogantes en torno a la implicación de los individuos infantiles en la creación del registro arqueológico, y más aún, en su papel social dentro del grupo humano (LILLEHAMMER, 1989). «Nińas y niños, están presentes en cualquier espacio de cualquier comunidad actual o pasada» (SÁNCHEZ ROMERO, 2010: 9); su crianza y educación son una motivación primaria en todas las sociedades (HOEBEL y WEAVER, 1985: 344). Siendo así, son escasos los estudios que afrontan el proceso de sociabilización de la infancia; niños y niñas han sido tradicionalmente relegados a un segundo plano, al amparo de la producción y función social de los adultos. Esto se debe al propio concepto de infancia que ha sido definido por la Modernidad. La infancia que concebimos en la actualidad es una construcción del siglo XIX de la clase media occidental, que identifica ésta con un período de juego y aprendizaje (CUNNINHAM, 1996). Se percibe la niñez por oposición a la edad adulta; viendo al niño/a como dependiente, inocente y necesitado de protección y socialización antes de que pueda participar completamente en el mundo social y cultural de los adultos (KAMP, 2001a y b). Este hecho es causa directa de la invisibilidad de la infancia en la Arqueología (CHAPA, 2003); un mundo visionado (e interpretado) por adultos a partir de un precario registro arqueológico.

Es por ello que su abordaje debe partir de una conciencia crítica, ya que la definición de infancia responde a una construcción cultural y no puede ser naturalizada para cualquier comunidad humana: «la infancia es un concepto dinámico y fluido que

3 «Las tendencias post-procesuales (...) han hecho hincapié en la importancia del individuo como actor dinámico en la configuración del lenguaje cultural, abriendo la puerta a la consideración de grupos sectoriales, y desarrollando especialmente los estudios de género. De hecho, la reflexión sobre el mundo infantil ha sido en muchos casos una consecuencia de la atención prestada al universo femenino dentro de cada unidad social» (CHAPA, 2003: 117). 
se mueve entre lo biológico y lo cultural con variaciones e interpretaciones que cambian a través del tiempo y del espacio" (NÁJERA et al., 2010: 70). Cada sociedad tiene su propia definición de categoría de edad para la infancia, si bien a grandes rasgos parece inscribirse entre el nacimiento y la edad adulta, momento en el que a la producción se une la capacidad de reproducción del individuo. Así, la categoría de edad, igual que la de género, está socialmente constituida (SÁNCHEZ ROMERO, 2007: 26).

Los estudios referidos a los individuos infantiles como creadores de registro arqueológico se han centrado en la búsqueda de evidencias de aprendizaje de la talla lítica (TIXIER et al., 1976; PIGEOT, 1987; HOCSMAN, 2006), la interpretación de los juguetes (POLITIS, 1998; 1999; CRAWFORD, 2009; KAMP, 2010), los estudios de huellas cerámicas como ejemplo de manufactura infantil (KAMP, 2001b)... y se han desarrollado, mayoritariamente, en el ámbito funerario (OLÀRIA, 2008; GIBAJA et al., 2010; DE MIGUEL, 2010). Sin embargo, existen otras "huellas» que se vinculan a un ámbito específico del registro arqueológico como es el espacio cavernario, a través del que puede ser interpretada la presencia (restos óseos) o transición de individuos infantiles (huellas de pies y manos), así como su participación en la ejecución (manos impresas) o contemplación del grafismo subterráneo. Este último elemento (el arte) permite reconstruir la representación que de la infancia tiene un grupo humano determinado (pinturas y grabados de individuos infantiles), en este caso, las sociedades paleolíticas en el continente europeo.

\section{METODOLOGÍA Y PROBLEMÁTICA}

En el espacio interior cavernario, el registro arqueológico se compone de pisadas, marcas, huellas, manos impresas, restos óseos y representaciones gráficas cuya forma y tamańo mensurable posibilita su análisis e interpretación. El primer problema al que nos enfrentamos es cómo deducir de su análisis que nos encontramos ante huellas (de manos y pies) infantiles. Los estudios en esta línea son numerosos. Centrados en cálculos matemáticos (SAHLY, 1966) y contrastados mediante la experimentación (LORBLANCHET, 1995; RIPOLL et al., 1999: 91-92) determinan una relación estadística entre el tamańo de las manos y los pies respecto a la altura (edad) del individuo (VALLOIS en BÉGOÜEN Y BREUIL, 1958; PALES, 1976; GARCIA, 2005: 105; BRŮŽEK et al., 2012: 1198). No obstante, son numerosas las variables que pueden alterar esta relación, tales como la superficie, la perpendicularidad del soporte (en el caso de las manos impresas sobre la pared rocosa), la densidad del suelo o su grado de deslizamiento.

Similar problemática encontramos en la identificación de individuos infantiles en las representaciones gráficas paleolíticas. Los análisis al respecto se han centrado en 
las manos impresas sobre las paredes de las cavidades y las figuraciones de individuos infantiles. Para la primera de ellas, la problemática ya ha sido expuesta y se basa en las posibles alteraciones de la superficie; en la segunda, el análisis morfológico y analógico de las representaciones posibilita su interpretación como figuras infantiles, muchas veces potenciada por el contexto narrativo de un conjunto gráfico ${ }^{4}$. Son muy escasas las representaciones documentadas en el arte paleolítico como 'infantiles', más aún si atendemos al porcentaje que éstas representan en relación al global de la muestra gráfica de manos y motivos humanos. Su atribución se debe a criterios fisiológicos y anatómicos tales como el tamaño de las representaciones, la presencia de un vientre hinchado, extremidades cortas o una marcada desproporción de la cabeza respecto al resto del cuerpo (PALES et al., 1976; DUHARD, 1993). Cuando la figura presenta detalles precisos, éstos pueden resultar de ayuda, como la forma abultada de la frente, el tamaño de la nariz, la identificación del cordón umbilical o la posición fetal o flexionada.

La identificación de enterramientos infantiles, por otra parte, está condicionada por su carácter intencional (HENRY-GAMBIER, 2008: 40), por la falta de una documentación rigurosa en las excavaciones antiguas y por su efímera materialidad. No obstante es llamativa, dada la precaria naturaleza de conservación de los frágiles huesos infantiles, la supervivencia de estos restos en el registro arqueológico; como se constata en el ámbito cantábrico (DE BLAS, 1997: 161). Desde la consciencia de la fragmentación del registro funerario paleolítico, abordamos la presente investigación. Para ello, se ha elaborado una base de datos de los restos óseos susceptibles de ser considerados como enterramientos, con el objetivo de reunir una muestra lo suficientemente significativa de sepulturas infantiles datadas en el Paleolítico superior europeo. No obstante, existen también restos óseos «descontextualizados» o en posición secundaria que ofrecen otro tipo de información acerca de los individuos infantiles y actividades realizadas en torno a su fallecimiento, como puede ser un cráneo trepanado hallado en Rochereil (VALLOIS, 1971), una mandíbula con marcas de una posible antropofagia (¿infanticidio?) en Lortet (BOIVIN, et al., 1981), huesos posiblemente con trazos de descarnación en l'Abri Lachaud (MAY, 1986: 56-57), un conjunto de restos óseos quemados junto a osamentas animales en una de las sepulturas de Dolni Vestonice (MAY, 1986: 95), restos óseos manipulados en Brillenshöhle y en Maszycka (OLÀRIA, 2008: 425 y 437), o un fragmento de una mandíbula infantil documentada en el Camarín de las Ciervas de Torneiros y atribuida a un individuo femenino (FERNÁNDEZ ALGABA et al., 2009). Todo ello condiciona la calidad del registro arqueológico con el que contamos, si bien no justifica la invisibilidad de dicho objeto de estudio en la Arqueología paleolítica tradicional.

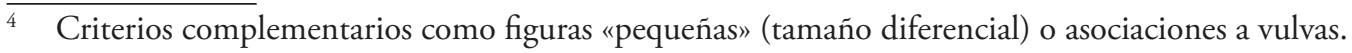




\section{CORPUS DE DATOS}

\subsection{Representaciones Parietales y MUebles}

Se han registrado hasta la fecha 32 figuraciones de posibles antropomorfos infantiles (tabla 1) que responden a los criterios expuestos en el apartado metodológico (vid. supra). De forma mayoritaria éstas se encuentran sobre plaquetas o losas muebles y han sido atribuidas por contexto estratigráfico al Magdaleniense; a excepción de una posible representación de un feto hallada en un nivel arqueológico atribuido al tecnocomplejo gravetiense, en la cavidad de Grimaldi (DUHARD, 1996: 38) y de una representación de un joven sobre un fragmento del friso de Roc de Sers, atribuido al tecnocomplejo solutrense (TYMULA, 2002: 170). En cuanto a los formatos de representación, se trata de unidades completas 5 en las que se trazó la cabeza, el tronco y las extremidades (superiores e inferiores) aunque no abundan en detalles. Las grafías son descritas como individuos infantiles en base a su talla reducida, en relación a otras unidades gráficas localizadas en su contexto más inmediato (GUY, 2003: 360). También son identificados como individuos infantiles algunos formatos simplificados (mitad superior y mitad inferior) ${ }^{6}$, siendo en los primeros representativa la forma abultada de la cabeza (macrocefalia); al tiempo que los segundos responden a extremidades cortas (braquitipia), en posición replegada o curvada y determinación de la anatomía sexual poco desarrollada (en la gran mayoría son grafías femeninas). El segundo formato más numeroso es el de la cabeza. A ésta se reducen algunas grafías, como se constata en una plaqueta de La Marche en la que se han documentado 5 cabezas infantiles (PALES et al., 1976). Sus rasgos han sido ya enunciados: cabezas grandes, con la frente abultada, el cuello corto, occipucio prominente y pequeña nariz redondeada (DUHARD, 1996: 158).

En cuanto a su contexto de representación, se debe advertir que en escasas ocasiones, las representaciones infantiles aparecen aisladas o individualizadas. Normalmente se localizan formado parte de una composición más elaborada en la que se registran figuras femeninas (La Marche, ob.35 y 40; Gönnersdorf; Grimaldi; Laugerie-Basse), representaciones vulvares (Réseau Guy Martin, Saint-Cirq, La Font-Bargeix, Arcy-sur-Cure, Combarelles) u otras grafías de niños/as asociadas (La Marche, ob. 27 y fig. 66). Esta última ha sido interpretada como una posible escena de danza (AIRVAUX, 2001: 92); también en Levanzo (GRAZIOSI, 1962: 45) donde la figura más pequeña podría ser identificada como individuo infantil. Cabe destacar la asociación observada por uno de los firmantes (A.L.) entre las escenas materno-fi-

5 Se han documentado 15 unidades gráficas completas, procedentes de los yacimientos de La Vache, La Marche (5 individuos), Saint-Cirq, Mas d'Azil (2 individuos), Bruniquel, Brassempouy, Teyjat (3 individuos) y Roc de Sers (ver tabla 1).

6 Se han documentado 4 unidades gráficas simplificadas, procedentes de Fontanet, Laugerie-Basse, Levanzo y Chaffaud (ver tabla 1). 


\begin{tabular}{|c|c|c|c|c|c|c|}
\hline \multirow{2}{*}{ YACIMIENTO } & \multicolumn{2}{|c|}{ SOPORTE* } & \multirow{2}{*}{ UGs } & \multirow{2}{*}{ FORMATO } & \multirow{2}{*}{ EDAD } & \multirow{2}{*}{ BIBLIOGRAFÍA } \\
\hline & M & $\mathbf{P}$ & & & & \\
\hline LA VACHE & $\mathrm{x}$ & & 1 & COMPLETO & INF-2 & GUY, 2003: 360; DUHARD, 1996: 159 \\
\hline FONTANET & & $\mathrm{x}$ & 1 & $\begin{array}{l}\text { MITAD } \\
\text { INFERIOR }\end{array}$ & PREINF & VIALOU, 1986: 53; DUHARD, 1996: 153 y 159 \\
\hline $\begin{array}{l}\text { LA MARCHE (RÉSEAU } \\
\text { GUY MARTIN) }\end{array}$ & $\mathrm{x}$ & $\mathrm{x}$ & $\begin{array}{l}10 \\
+1\end{array}$ & $\begin{array}{l}\text { CABEZA (5) } \\
\text { COMPLETO } \\
(5) \\
\text { CABEZA+ } \\
\text { TRONCO }\end{array}$ & $\begin{array}{l}\text { PREINF, } \\
\text { INF-1 } \\
\text { INF-2 }\end{array}$ & $\begin{array}{c}\text { PALES y TASSIN, 1976; } \\
\text { DUHARD, 1996: } 158-162 ; 198 \\
\text { MÉLARD, 2008:145 y } 202 \\
\text { AIRVAUX, 2001: } 92-94,114 \text { y } 134 \\
\text { TOSELLO y FRITZ, 2005: } 22\end{array}$ \\
\hline SAINT-CIRQ & & $\mathrm{x}$ & 1 & COMPLETO & PREINF & $\begin{array}{l}\text { LEROI-GOURHAN, 1965: 47; MÉLARD, 2008: } \\
\text { 202; AIRVAUX, 1998: } 516 .\end{array}$ \\
\hline MAS D'AZIL & $\mathrm{X}$ & & 2 & COMPLETO & INF-1 & DUHARD, 1992: 295; CHOLLOT, 1964: 285 \\
\hline GRIMALDI & $\mathrm{x}$ & & 1 & CABEZA & PREINF & DUHARD, $1996: 38$ \\
\hline BRUNIQUEL & $\mathrm{x}$ & & 2 & $\begin{array}{l}\text { COMPLETO } \\
\text { MITAD } \\
\text { SUPERIOR }\end{array}$ & INF-1 & $\begin{array}{c}\text { DUHARD, 1996: } 159 \\
\text { PIETTE, 1907; CHOLLOT, 1964: } 467\end{array}$ \\
\hline \multirow[t]{2}{*}{ LAUGERIE-BASSE } & $\mathrm{x}$ & & 1 & ACÉFALO & INF-1 & DUHARD, 1993: 79 \\
\hline & $\mathrm{x}$ & & 1 & CABEZA & INF-1 & CALDWELL, 2012: 448, fig. 12 \\
\hline LA FONT BARGEIX & \multicolumn{2}{|c|}{$i$} & 1 & $\begin{array}{c}\text { CASI } \\
\text { COMPLETO }\end{array}$ & INF-1 & DELLUC y DELLUC, 2009: 639 \\
\hline GÖNNESDORF & $\mathrm{x}$ & & 1 & $c$ & PREINF & $\begin{array}{l}\text { SANCHIDRIÁN, 2001: 110; } \\
\text { DUHARD, 1996: 198-199; } \\
\text { BOSINSKI et al., 2001, taf.99, pla. 87a y b. }\end{array}$ \\
\hline BRASSEMPOUY & $\mathrm{x}$ & & 1 & COMPLETO & INF-1 & PIETTE, 1895: 147 \\
\hline ARCY-SUR-CURE & & $\mathrm{x}$ & 1 & $i$ & INDET & BAFFIER y GIRARD, 1988: 76-77 \\
\hline LEVANZO & & $\mathrm{x}$ & 1 & $\begin{array}{l}\text { MITAD } \\
\text { SUPERIOR }\end{array}$ & INDET & GRAZIOSI, 1962: 45 \\
\hline COMBARELLES & & $\mathrm{x}$ & 1 & $i$ & INDET & $\begin{array}{l}\text { CAPITAN et al., } 1924 \text { : 56, fig.53.1; } \\
\text { SACCASYN, 1947: 196, fig. } 93 \text {. }\end{array}$ \\
\hline CHAFFAUD & $\mathrm{x}$ & & 1 & $\begin{array}{l}\text { MITAD } \\
\text { SUPERIOR }\end{array}$ & PREINF & AIRVAUX, 2002: 23, fig.20 y 21. \\
\hline TEYJAT & $\mathrm{X}$ & & 3 & COMPLETO & INDET & SACCASYN, 1947: 58 , fig. 25 \\
\hline ROC DE SERS & & $\mathrm{x}$ & 1 & COMPLETO & ADO & TYMULA, 2002: 179, fig.109 \\
\hline
\end{tabular}

Tabla 1. Corpus de representaciones gráficas interpretadas como individuos infantiles.

liales y los soportes muebles; así como entre las escenas naturales o sexuales (vulva + individuo infantil) y los dispositivos parietales. Por último, algunas representaciones juveniles aparecen asociadas a animales, como se documenta en Fontanet y más claramente en La Vache, Mas d'Azil y Roc de Sers, donde se ha visto una posible escena de iniciación a la caza (TYMULA, 2002: 179). En ningún caso (tal vez en la «escena» de La Vache) se documenta la presencia de individuos adultos masculinos asociados (figura 1). Ello puede ser reflejo de la realidad social del grupo humano autor de las representaciones, en las que el cuidado, atención y sociabilización de los individuos infantiles descansa sobre el género femenino (como se materializada en Gönnersdorf), al cual se responsabiliza de la reproducción y producción social del grupo. No es de extrańar, por tanto, que las grafías reproduzcan un elemento constante y cotidiano. Por otra parte, las representaciones de varias figuras infantiles asociadas señalan la vivencia común de este subgrupo dentro de la sociedad paleolítica. 

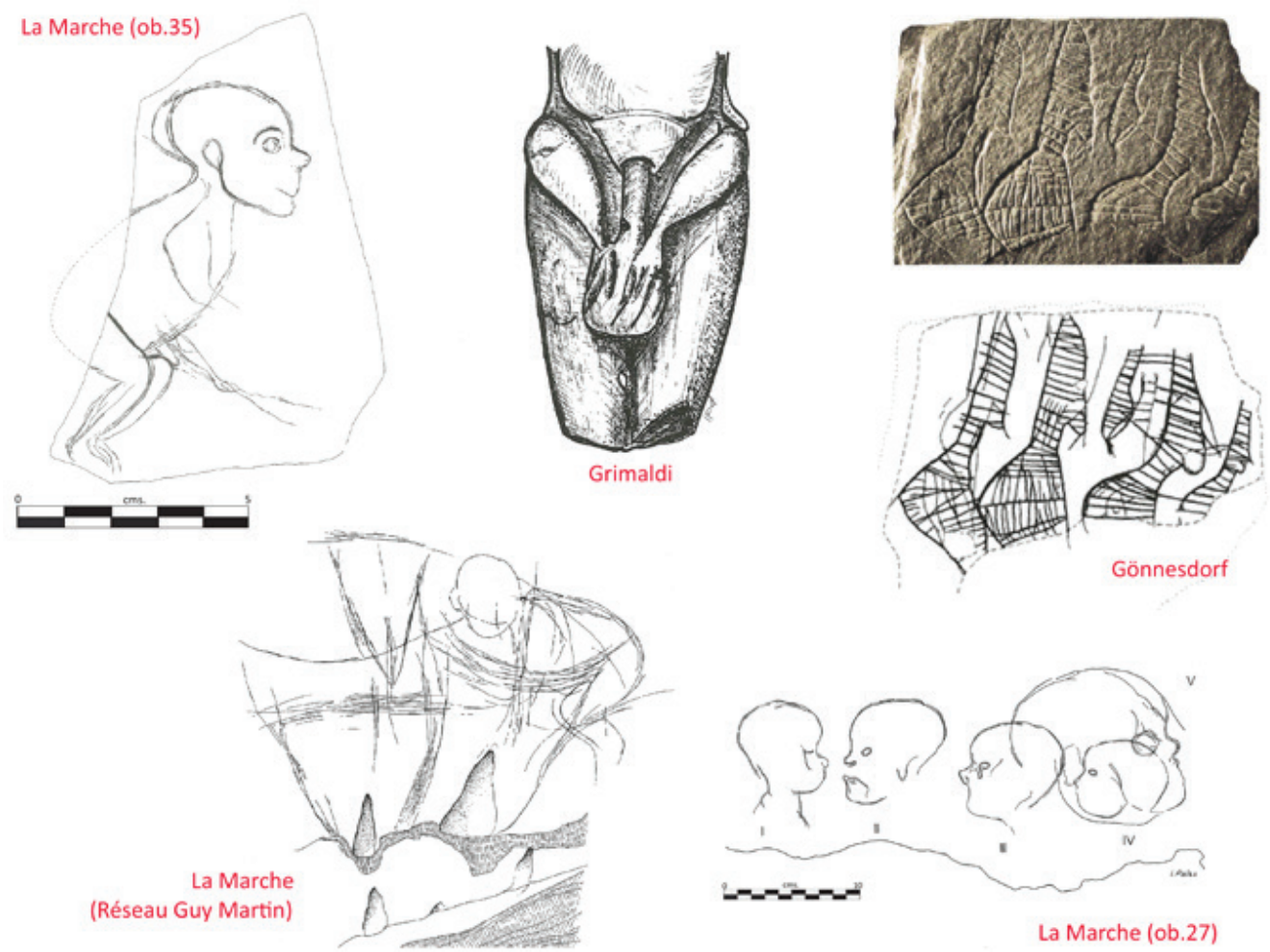

Figura 1. Ejemplos de representaciones de individuos infantiles en el arte paleolítico (parietal y mueble) europeo. La Marche (a partir de PALES, 1976: pl. 57 y 58; AIRVAUX, 2001: 114, fig. 96), Grimaldi (a partir de DUHARD, 1996: 38) y Gönnersdorf (a partir de BOSINSKI, 2001: 53, fig. 4).

\subsection{EnterRamientos}

La presente investigación ha documentado un total de 75 individuos infantiles en el registro funerario paleolítico europeo. En todo caso, se ha operado siempre con el número mínimo de individuos (NMI) identificado en cada enterramiento. La estimación de la edad de los restos óseos infantiles se ha efectuado en base al desarrollo dental y al grado de osificación del esqueleto (HENRY-GAMBIER, 2008: 403), estableciendo un rango de edad para cada uno de los sujetos documentados. Siguiendo a T. Chapa (2003: 16) se han tipificado 3 franjas de infancia que permiten la comparación y el análisis interpretativo del registro ( $v i d$. tabla 2) y que responden a una distinción antropológica vinculada con etapas básicas de crecimiento. La primera de ellas agrupa a los individuos de entre 1 y 7 ańos (INF-1); seguida de aquéllos que se estiman entre los 8 y 14 años (INF-2) y los que se encuentran entre los 15 y los 22 (ADO), cuya consideración como adolescentes o subadultos puede no ajustarse a la dinámica social de los 
grupos paleolíticos. Hemos distinguido, además, un subgrupo denominado PREINF, referida a los restos correspondientes a individuos neonatos, perinatales e inferiores al año ( $<1$ año), que tienen en su misma inmadurez la causa de muerte. A continuación, se ha establecido un quinto tipo en el que se engloban todos los restos de edad indeterminada (INDET) (tabla 2).

Si dejamos a un lado los individuos que se han documentado sin un contexto funerario seguro ( 5 individuos), es destacable la presencia de enterramientos individuales (16), seguido de enterramientos múltiples (12, en los que se han documentado 37 individuos infantiles) y enterramientos dobles ( 9 , con 13 individuos infantiles); así como la asociación establecida entre dichas formas de deposición y la edad de los individuos sepultados. Esta relación muestra el empleo de todo tipo de enterramientos para los individuos pertenecientes a los grupos de edad INF-1 e INF-2; destacando, por el contrario, la importancia cuantitativa de los individuos menores de 1 año en los enterramientos múltiples (19\% de la muestra). Esta realidad se explica por la presencia de numerosos restos prenatales o perinatales, que implican la muerte del feto o recién nacido y de la madre durante el parto o con anterioridad a éste a causa de una malformación o complicación de tipo obstétrico (vid. gráfico 1). Es por ello que en dichos enterramientos los individuos aparecen asociados a sujetos adultos femeninos, como se ha documentado en Cro-Magnon $^{7}$ (HENRY-GAMBIER, 2002), Abri Pataud ${ }^{8}$ (MAY, 1986: 59-60) y en el yacimiento de Dolni Vestonice 9 (OLÀRIA, 2008); cuyos contextos funerarios se adscriben al tecnocomplejo gravetiense.

En cuanto a la presencia de ajuar o de otros elementos asociados a los enterramientos, tan sólo se han documentado 18 casos de estudio (24\% de la muestra), siendo 11 de ellos referidos a sepulturas individuales. Este dato sugiere la participación de los individuos infantiles en la vida de la comunidad desde el momento de su nacimiento. Teniendo en cuenta la importancia de los procesos de producción y reproducción de una sociedad cazadora-recolectora, el nacimiento de un individuo se comprendería como «bien» del grupo (un bien para la sociedad), sobre el que recaería la responsabilidad de su protección y sociabilización hasta convertirse en un miembro productivo para ésta. Dicha dinámica puede ser muy significativa si tenemos en cuenta que sólo dos casos de

$7 \quad$ El enterramiento hallado en el sitio de Cro-Magnon alberga 5 individuos, dos varones adultos, un adulto indeterminado, una mujer adulta y un individuo infantil, cuya relación no ha sido determinada (HENRY-GAMBIER, 2002).

8 En el posible enterramiento de Abri Pataud, aparece por dos veces la asociación de restos de una mujer con un recién nacido ¿inmolación de niño con su madre difunta, o muerte del niño al morir su madre? (MAY, 1986: 59-60).

9 La sepultura 3 de este yacimiento alberga un enterramiento múltiple compuesto de dos hombres adultos, una mujer y un recién nacido. Posiblemente se trate de una muerte postparto, ya que la mujer presenta una deformación de la pelvis y un depósito de ocre entre sus piernas (OLÂRIA, 2008). 


\begin{tabular}{c|c|c} 
TIPIFICACIÓN & RANGO DE EDAD & N.M.I. (TOTAL: 96) \\
\hline PREINF & $<1$ año & 15 \\
\hline INF-1 & $1-7$ años & 23 \\
\hline INF-2 & $8-14$ años & 20 \\
\hline ADOLESCENTE & $14-22$ años & 6 \\
\hline INDETERMINADO & Indeterminado & 11
\end{tabular}

Tabla 2. Cuantificación de los enterramientos infantiles agrupados según su rango de edad.

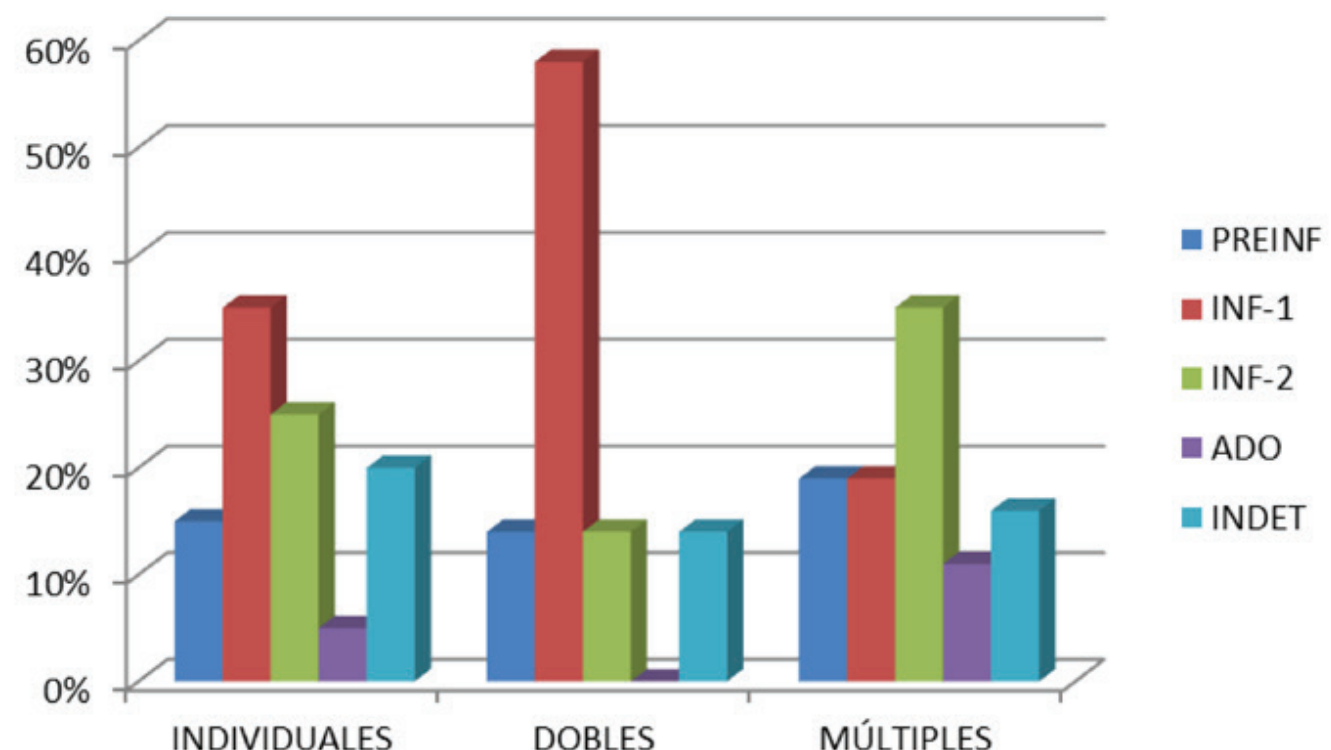

Gráfico 1. Reparto porcentual de los grupos infantiles en relación con el tipo de enterramiento.

los reseńados (Le Figuier y Abri de Peyrugues) se atribuyen a un momento posterior al Último Máximo Climático ${ }^{10}$ (ca. $\left.18000 \mathrm{BP}\right)$. En las etapas cronológicas precedentes «la progresiva ocupación de nuevos territorios y la competencia desigual con una humanidad diferente -los neandertales- parecen conformar una situación adecuada para que surja una ideología que prime el incremento de efectivos humanos propios y la ampliación de las redes sociales de nuevos pobladores» (GONZÁLEZ MORALES, 2007: 63). Es por ello, que cobra sentido la presencia de enterramientos infantiles individuales con

10 Este dato es muy significativo ya que del corpus global de enterramientos analizado, 51 registros (53\%) pertenecen a momentos anteriores del UMC, 42 para los enterramientos atribuidos al tecnocomplejo epigravetiense o magdaleniense (44\%); y en tan sólo 4 casos (3\%), la cronología es dudosa o poco precisa. 


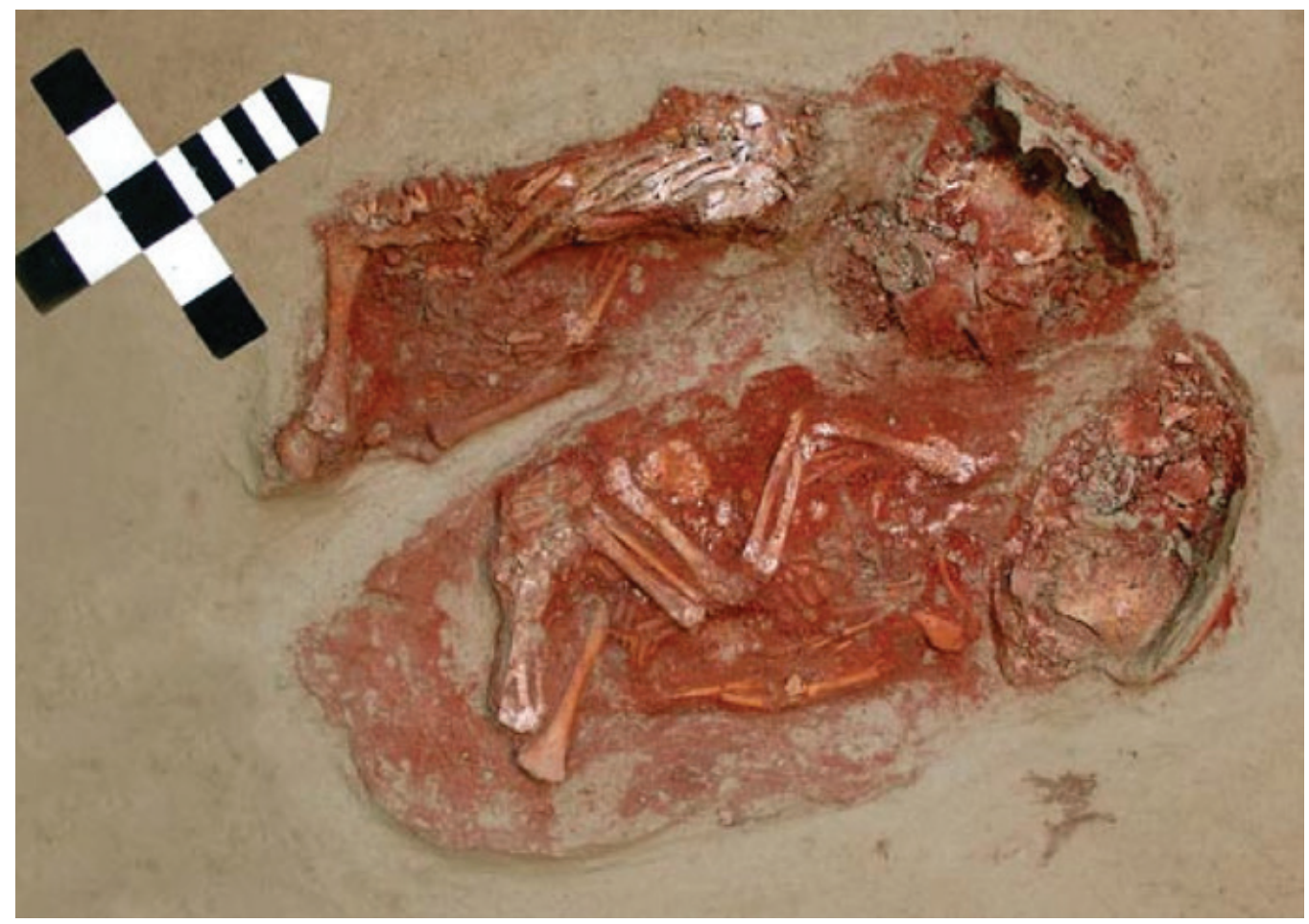

Figura 2. Enterramiento de dos individuos infantiles (PREINF) hallado en Krems-Wachtberg (Austria). (9/04/2013): <http://www.20minutos.es/noticia/172817/0/paleolitico/superior/ bebes/>).

ajuares que reconocen la importancia social del miembro fallecido para la comunidad a través de la introducción junto al cadáver de conchas (Arene Candide; Grotte Marronnier), dientes perforados (Paglicci), colgantes de marfil (Arene Candide), láminas de sílex, brazaletes, cuentas de marfil (Malta), e incluso un elaborado tocado compuesto de 150 dientes de zorro perforados sobre la cabeza de un niño de unos 6 años, que ha sido documentado en Kostienki-15 (BINANT, 1999; IAKOVLEVA, 2008) (figura 2).

La dificultad de determinación del sexo en los individuos infantiles no permite una aproximación mayor. Tan sólo se ha podido identificar éste en 7 individuos, con valores muy equilibrados entre ellos ( 3 femeninos y 4 masculinos). Toda inferencia o interpretación sería absurda, dada la escasez de datos al respecto.

De todo lo dicho se puede deducir que los individuos infantiles, en contra de la visión tradicional, tienen una importancia destacable en la sociedad paleolítica, bien como potenciales miembros productivos y reproductivos de ésta (en el caso de los preinfantes; PREINF) así como generadores de registro arqueológico. 


\subsection{LAS IMPRONTAS DE NIÑOS EN EL REGISTRO ARQUEOLÓGICO}

Las improntas de niños en el registro arqueológico son percibidas desde una doble óptica, en función de que éstas hayan sido o no intencionales. Esta clasificación resulta muy útil para establecer categorías de análisis diferenciadas: por un lado, encontramos las huellas no intencionales (MOURE et al., 1984/85: 19), es decir marcas hechas con la mano, el dedo, la rodilla, el torso o los pies sobre superficies blandas arcillosas a las que se les suele otorgar cierto carácter fortuito (CLOTTES y COURTIN, 1994: 63) o se las denomina directamente como huellas de tránsito, por ejemplo en Niaux (CLOTTES Y SIMONET; 1972b: 27; GARCÍA et al., 1990), en Pech Merle (DUDAY y GARCÍA, 1983: 214, cita 9) o en Chauvet (GARCÍA, 1999). Esta diferenciación, aunque acrítica, resulta útil para diferenciarlas de las improntas de manos que requieren cierto grado de preparación y el uso de otros útiles empleados en la ejecución de los grafismos, lo cual favorece su interpretación como acción intencional. Desde el comienzo de los estudios de arte paleolítico como disciplina arqueológica se registraron en el elenco de manifestaciones gráficas, un conjunto de manos impresas (en positivo o negativo) sobre soporte parietal. Tradicionalmente las manos han formado parte del conjunto de signos gráficos, no obstante, M. Groenen les da una categoría independiente (GROENEN, 2000: 46). La mano humana no es un elemento simbólico o un grafismo de interpretación desconocida. Sabemos interpretar su adscripción antropomorfa y más aún se puede aproximar la edad del autor/a de dicha grafía, ampliando así nuestros conocimientos acerca de la sociedad y su relación con el hecho gráfico paleolítico.

En cuanto a las huellas de pies infantiles, se ha recogido un corpus de 7 conjuntos de pisadas (tabla 3). Algunas de ellas pueden pertenecer a una misma persona, mientras que otras parecen ser complementarias de un transcurso o recorrido que se realizó en el interior de la cavidad. No obstante, hemos de destacar que la presencia de estas huellas en el suelo, conservadas en la actualidad, las convierte en elementos un tanto «anecdóticos». La conservación de una huella humana en la superficie cavernosa indica que sobre ella no hubo una nueva actividad porque un nuevo uso del espacio la hubiera destruido; "ont plus de chances de parvenir jusquà nous, tout simplement parece que les jeunes empruntent des intinéraires atypiques, où leurs traces risquent moins d'être effacées par la multiplicité de passages» (GARCÍA, 1999: 3). Luego, su presencia tan sólo responde a un único acontecimiento no extrapolable o generalizable a un uso frecuente del espacio.

\subsubsection{Huellas infantiles: pies y manos sobre arcilla / suelo (tránsito y participación en actividades de la comunidad)}

Las huellas de pies y manos conservadas proceden de diversas acciones (presencia, tránsito o permanencia) que han quedado impresas en superficies blandas de la 


\begin{tabular}{|c|c|c|c|c|c|}
\hline YACIMIENTO & UBICACIÓN & IMPRONTA & NMI & EDAD & BIBLIOGRAFÍA \\
\hline PECH- MERLE & Le réseau & Huellas de pies & 1 & $\begin{array}{l}\text { Niño, } \\
\text { adolescente o } \\
\text { adulto de pie } \\
\text { grácil }\end{array}$ & DUDAY y GARCÍA, 1983 \\
\hline CHAUVET & $\begin{array}{l}\text { Galerie des } \\
\text { Croisillons-Salle } \\
\text { du Cråne }\end{array}$ & Huellas de pies & 1 & $\begin{array}{l}\text { Niño de } 1^{\prime} 30 \text { m } \\
\text { (8 an̂́os) }\end{array}$ & $\begin{array}{l}\text { GARCÍA, 1999; GARCÍA, 2005: } \\
105 .\end{array}$ \\
\hline \multirow[b]{2}{*}{ FONTANET } & Réseau Wahl & Huellas de pies & & & CLOTTES, 1973: 489 \\
\hline & $\begin{array}{l}\text { Réseau Wahl (en } \\
\text { un escondrijo) }\end{array}$ & Manos y pies & & & $\begin{array}{l}\text { CLOTTES, 1973: } 490 \text {; } \\
\text { CLOTTES, 1993: } 62 \text {, fig 18; } \\
\text { CLOTTES Y COURTIN, 1994: } \\
63\end{array}$ \\
\hline \multirow{2}{*}{$\begin{array}{l}\text { TUC } \\
\text { D'AUDOUBERT }\end{array}$} & $\begin{array}{l}\text { Galerie des } \\
\text { empreintes }\end{array}$ & Huellas de pies & 1 & 7-9 años & $\begin{array}{l}\text { BÉGOÜEN y BREUIL, 1958: 99; } \\
\text { PALES, 1976: } 46\end{array}$ \\
\hline & Salle des talons & Huellas de pies & & 9-12 años & $\begin{array}{l}\text { BÉGOÖEN y BREUIL, 1958: } \\
\text { 100; PALES, 1976: } 46\end{array}$ \\
\hline NIAUX & $\begin{array}{l}\text { Le Réseau René } \\
\text { Clastres. }\end{array}$ & Huellas de pies & 3 & $\begin{array}{l}\text { menos de } 10 \\
\text { ańos }\end{array}$ & $\begin{array}{l}\text { PALES, 1976b: } 85 \text {; CLOTTES Y } \\
\text { SIMONET, 1972a; CLOTTES Y } \\
\text { SIMONET, 1972b; CLOTTES, } \\
\text { 1972b; }\end{array}$ \\
\hline COSQUER & Sector 102 y 106 & 3 Manos & & Niños & CLOTTES et al., 2005: 17 \\
\hline ARDALES & $\begin{array}{l}\text { Sector IV Galería } \\
\text { del Calvario }\end{array}$ & 1 mano & & 7-9 ańos & CANTALEJO et al., 2006: 361 \\
\hline COMBARELLES & Panel 71 & Cuatro dedos & 1 & $\begin{array}{l}\text { ¿Niño? Mano } \\
\text { pequeña }\end{array}$ & $\begin{array}{l}\text { CAPITAN, et al., 1924: 61, fig. } \\
58\end{array}$ \\
\hline
\end{tabular}

Tabla 3. Corpus de huellas de transición asociadas a la actividad de individuos infantiles.

cavidad (suelos, repisas y soportes en disposición vertical) hasta la actualidad. Su carácter intencional ha sido admitido convencionalmente por los especialistas (BÉGOÜEN y BREUIL, 1958: 98; BELTRÁN et al., 1973), enmarcados en un contexto postmoderno en el que se radicalizó la percepción del mundo simbólico y ritualizado (CLOTTES y LEWIS-WILLIAMS, 1996). Actualmente, parece advertirse una propuesta simétrica $^{11}$ en la que se analizan las impresiones, en una primera instancia, como dato arqueológico que señala la presencia de individuos infantiles en la cavidad, pudiendo obtener de su estudio tipométrico el número de individuos autores de las mismas, la posible diacronía del registro (CLOTTES y SIMONET, 1972b: 31); la reconstrucción de su estatura (PALES, 1976: 85); e incluso, el recorrido o tránsito que se desarrolló en el interior de la cavidad (CLOTTES, 1972b: 29; GARCIA et al., 1990: 168). En segundo lugar, se proponen las interpretaciones, que conservan aún una profunda dicotomía entre el interior y el exterior de las cavidades, lo sagrado y lo profano, lo ritual y lo cotidiano... carentes de horizontes de subjetividad (CRIADO, 2012) que

$\overline{11}$ La base teórica es la denominada Arqueología simétrica, en la que tras la valoración radical del plano simbólico o ritual de la materialidad arqueológica, se vuelve a revalorizar el dato arqueológico, positivo y cuantificable, concretando en una simetría teórica entre la interpretación y el análisis positivista. 

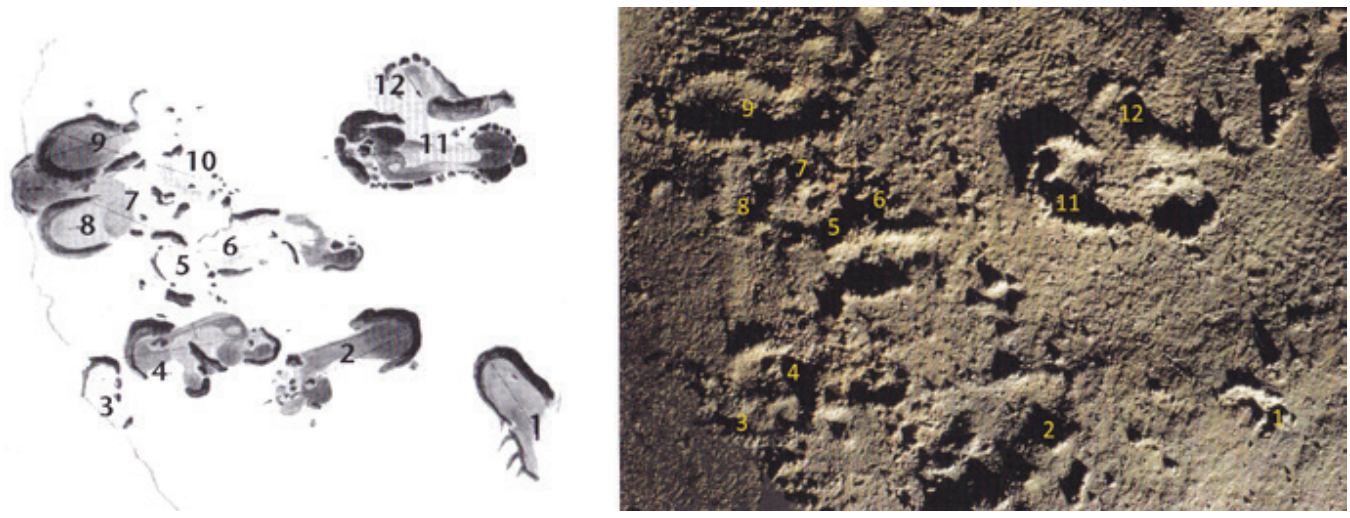

Figura 3. Huellas humanas (¿infantiles?) documentadas en Galerie de l'Ours (Pech-Merle).

Fotografía y calco de M. García (a partir de LORBLANCHET, 2010: 176).

obtenidos a partir de estudios etnográficos o etnoarqueológicos, permitan explicaciones ad hoc (VILÁ, 2006).

El corpus registrado para la presente reflexión consta de 8 cavidades (Pech-Merle, Chauvet, Fontanet, Tuc d'Audoubert, Niaux, Cosquer, Ardales y Combarelles) en las que se han documentado improntas de pies y manos, atribuidas a individuos infantiles. En alguno de los casos estudiados se ha identificado un único individuo autor de las huellas (Pech-Merle y Chauvet) pudiendo atestiguar así su presencia y tránsito por la cavidad (figura 3). Se trata, en ambas cavidades, de huellas de un individuo pre-adulto (en torno a los 8-10 ańos), en base al tamaño de la impresión $(21,4$ x 9,2cm. de media en Chauvet; GARCÍA, 2005: 105) y del cálculo de su altura (1,30m.). A este mismo segmento de edad parecen corresponder la mayor parte de huellas documentadas: en Tuc d'Audoubert (las dimensiones máximas alcanzan los $28 \mathrm{~cm}$.) se ha propuesto un margen de edad de entre 7 y 12 ańos (altura aproximada de 1,20m.; BEGOÜEN y BREUIL, 1958: 99) y en Niaux, inferior a los 10 años (CLOTTES y SIMONNET, 1972b: 29). Las posibles edades reconocidas han supuesto un cambio explicativo en cuanto a la vinculación de estas huellas con rituales iniciáticos asociados a adolescentes (PALES et al., 1976: 46-47).

\subsubsection{Manos de niños/as impresas (intencionalidad gráfica)}

La impresión de manos (en positivo o negativo) es un hecho constatable en el registro gráfico paleolítico europeo, pero este motivo trasciende el tiempo y el espacio 
de esta investigación, pudiendo ser documentado en el presente ${ }^{12}$; así como en América, África y Australia; siendo «un símbolo único que se repite» (RIPOLL et al, 1999: 97) sin que se pueda inferir un único significado. La representación de manos en el arte paleolítico ha ocupado la mente de numerosos investigadores (UTRILLA, 2005; UTRILLA y MONTES, 2007) quienes han denunciado la inexistencia de una explicación global. El contexto de la "oscura caverna» ha sido el elemento esencial sobre el que se han basado las interpretaciones, desde la posesión del submundo (CLOTTES et al., 2005: 20) a los rituales chamánicos (CLOTTES y LEWIS, 2001: 90-91), símbolos astrales (LACALLE, 1996), códigos gestuales (LEROI-GOURHAN, 1967; BAFFIER Y GIRAUD, 1998; RIPOLL et al., 1999) o ceremonias de iniciación. Sin embargo, estas propuestas interpretativas se construían sobre la forma, color o técnica gráfica de las representaciones, sin tener en cuenta su autoría. El carácter infantil de algunas de ellas nos invita a reflexionar acerca del papel que los niños y niñas tenían en el hecho gráfico, así como su participación en la cotidianeidad social del grupo. Contamos con dos corpus fundamentales de manos impresas en el registro parietal paleolítico franco-cantábrico (RIPOLL et al., 1999; GROENEN, 2000: 49) que elevan a 507 manos negativas y 22 positivas, el primero; y un total de 495 manos impresas, el segundo. Hemos recogido datos acerca de manos infantiles en 11 cuevas (tabla 4). Su número es incierto pero, tal y como percibíamos en las representaciones de huellas de manos y pies infantiles, el porcentaje es mínimo; aunque acredita, ya no la presencia de individuos infantiles, sino su participación consciente en el hecho gráfico de la comunidad de la cual forma parte. Algunas de las representaciones documentadas se atribuyen a individuos de muy corta edad, de unos 3-4 años o incluso bebés en el caso de La Fuente del Trucho (UTRILLA, 2005: 343; UTRILLA, et al., e.p.) (Figura 4) o de Gargas (SAHLY, 1966; BARRIÈRE, 1975: 201; FOUCHER et al., 2007: 81). La coincidencia de éstas con la menor altura respecto al suelo nos permite ańadir un dato de importancia interpretativa. Así se ha documentado en La Garma, donde «en la zona VIII se parecía un importante panel con manos pequeñas en amarillo a menor altura y peor realizadas técnicamente que las de tamaño mayor y más altas» (ARIAS et al., 2001: 53) o en Maltravieso (mano 38 y 67 en RIPOLL et al., 1999: 139 y 158) para las que se ha calculado la altura del autor/a en 1,49 y $1,37 \mathrm{~m}$ respectivamente. Su conservación incompleta ha generado numerosas hipótesis que no pueden ser corroboradas, formando parte del horizonte de inteligibilidad de la arqueología prehistórica. No focalizaremos el trabajo en el porqué de dichas manifestaciones, sino en la posibilidad de inferencia que su presencia en el registro gráfico supone ${ }^{13}$ con respecto a la participación de los individuos en las actividades cotidianas o simbólicas de la comunidad.

12 «Las cavernas y abrigos rocosos de Chiapas siguen siendo utilizados en la actualidad para diversas ceremonias» y contienen representaciones de manos en positivo (CERRADA, 2007: 50).

13 "Il paraît évident que des enfants ou des jeunes ont participé aux activités réalisées par les hommes paléolithiques à cet endroit» (BALBÍN et al., 2002: 574). 


\begin{tabular}{|c|c|c|c|}
\hline CUEVA & TÉCNICA & NMI & BIBLIOGRAFÍA \\
\hline FUENTE DEL TRUCHO & negativas & Indeterminado & UTRILLA y MONTES, 2007; UTRILLA et al, e. p. \\
\hline GARGAS & & 5 niños, 2 de bebé & $\begin{array}{l}\text { SAHLY, 1966; LEROI-GOURHAN, 1967; BARRIÈRE, } \\
\text { 1975; BARRIĖRE, 1984; FOUCHER et al., } 2007\end{array}$ \\
\hline FUENTE DEL SALÍN & & 3 & $\begin{array}{l}\text { MOURE et al., 1984-1985; BOHIGAS y SARABIA, } \\
\text { 1988; VALLE y SERNA, 2002: } 58 \text { y 60-61 }\end{array}$ \\
\hline MALTRAVIESO & negativas & 2 & RIPOLL et al., 1999 \\
\hline BAYOL & & 1 & DROUOT, 1984: 324 \\
\hline CASTILLO & & Indeterminado & $\begin{array}{l}\text { ALCALDE DEL RíO, et al., 1911: 120; RUIZ } \\
\text { REDONDO, 2010: } 25\end{array}$ \\
\hline ¿LA LASTRILLA? & positiva & ¿1? & MOLINERO, $2002: 310$ y fig. 8 \\
\hline ARCY-SUR-CURE & & 3 & BAFFIER y GIRAUD, 1998: 71 \\
\hline TIBIRAN & & Indeterminado & CLOT, 1984 \\
\hline LA GARMA (zona VIII) & negativas & indeterminado & ARIAS CABAL et al., 2001: 53 \\
\hline COMBARELLES & negativa & 1 & CAPITAN, et al., 1924: 47 y 49, fig. 41 \\
\hline
\end{tabular}

Tabla 4. Corpus de manos impresas atribuidas a autores infantiles.

\subsubsection{Discusión y síntesis}

Muchas de las representaciones artísticas identificadas como infantiles por los/as autores/as no pueden ser recogidas sin discusión.

En primer lugar, los criterios anatómico-fisiológicos nos ofrecen una serie de indicios para identificar una figura como «infantil», aunque determinados rasgos, como la indeterminación sexual, asimilados como propios de los individuos infantiles (DUHARD, 1996: 17), han de ser tratados con cautela. Otros aspectos, como el tamaño diferencial, la posición fetal o replegada y la asociación con vulvas, son criterios secundarios que han de ser tenidos en cuenta con mucha precaución.

Por otro lado, en muchos de los casos los calcos merecerían una revisión a fondo in situ, pues se trata de trabajos antiguos con metodologías de registro superadas en la actualidad. De hecho, uno de los rasgos descritos como cordón umbilical (DUHARD, 1996: 49) en el individuo de Saint-Cirq, puede ser igualmente atribuido a un pene de largura excepcional (DAMS, 1980: 62); al tiempo que las representaciones asociadas a vulvas de Combarelles pueden ser tanto figuras esquemáticas femeninas (BOSINSKI, et al., 2001) como elementos no-figurativos... A falta de un análisis in situ, se han recogido en este trabajo, todos los casos referidos a «infantiles» en la bibliografía especializada.

Las grafías identificadas o interpretadas como infantiles, tal y como ocurre en todas las sociedades históricas (VVAA, 2006), son realmente escasas. No obstante, éstas deben responder a la imagen en que las sociedades paleolíticas representaron a sus nińos/as. Las 


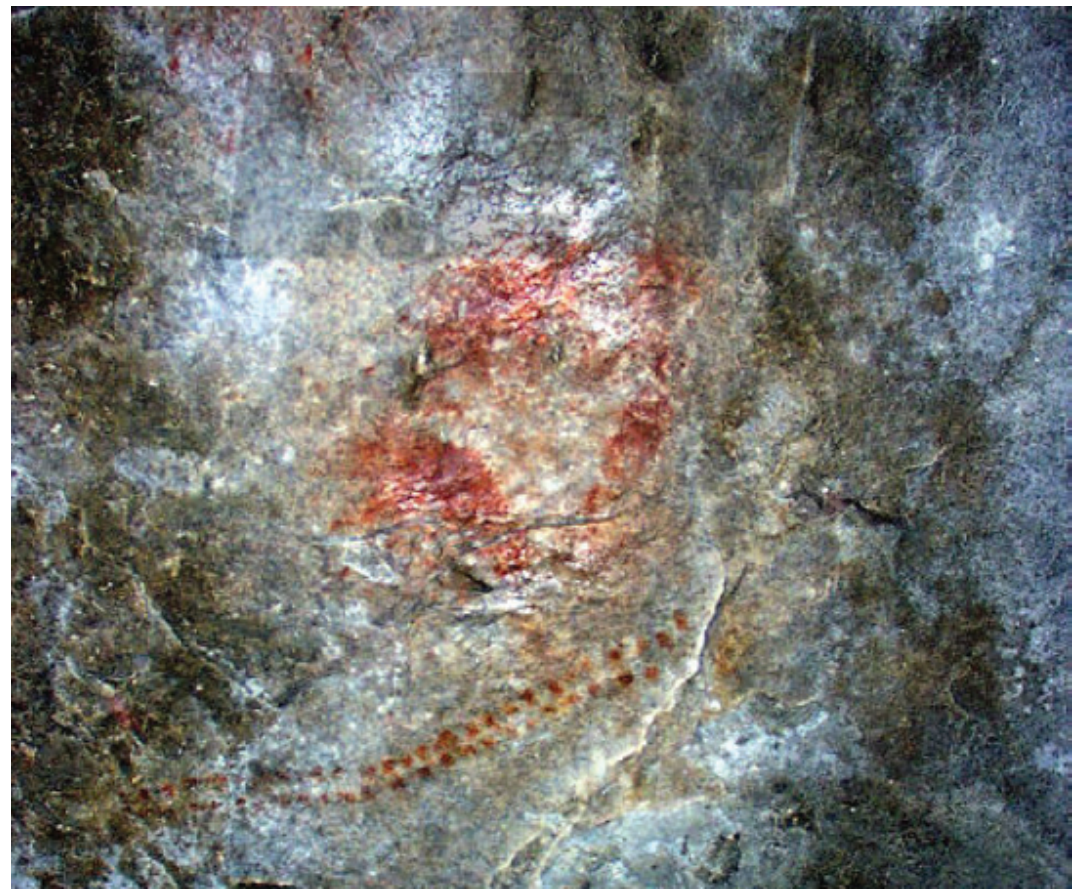

Figura 4: Mano infantil en negativo documentada en la Fuente del Trucho (fotografía gentileza de P. Utrilla).

grafías recogidas presentan diferentes formatos y asociaciones (junto a otros niños/as, mujeres, vulvas y animales) y hacen referencia a actividades sociales, económicas (de producción y de reproducción) y tal vez simbólicas (AIRVAUX, 1998; TOSELLO Y FRITZ, 2005). La relación de las mujeres con los recién nacidos se observa también en los enterramientos de Cro-Magnon, Pataud, Ostuni (HERY-GAMBIER, 2008: 404) y en una figurita de marfil posiblemente relacionada con el perinatal de Wilczyce e interpretada como una representación femenina encinta (BORÓN et al., 2012: 1389-1390). Los enterramientos de recién nacidos, los adornos, los vestidos de La Madeleine (VANHAEREN Y D'ERRICO, 2001), de Grotte des Enfants (OLÀRIA, 2008; BINANT, 1991: 60), de Sungir (VANHAEREN y D'ERRICO, 2001; MAUREILLE, 2004: 84) o de Malta (GOLOMSHTOK, 1993), nos hablan no sólo de la existencia de un sentimiento hacia ellos, sino del papel que los adultos otorgan a los individuos infantiles dentro de las sociedades paleolíticas, incluso en el mundo de las creencias y en los mitos, si aceptáramos la famosa interpretación de S. Reinach sobre los embriones infantiles mitológicos (Ratapás) en Teyjat. Explicaciones quizás, al «misterio del nacimiento» (WELTÉ y LAMBERT, 2005: 725) humano. Por otra parte, las sepulturas de adolescentes en Sungir ofrecen objetos ligados a la caza (IAKOVLEVA, 2008: 68), quizás reflejo de una temprana iniciación cinegética que pudiera vincularse con las interpretaciones de las representaciones artísticas de Roc de Sers y Mas d'Azil. 
Pero los niños son también sujetos activos en el registro arqueológico paleolítico como atestiguan las manos y las pisadas en el interior cavernario (LEROI-GOURHAN, 1967: 118; LEROI-GOURHAN, 1971: 307). Las manos de bebés de la Fuente del Trucho y Gargas, así como las manos de niños muy pequeños, no pueden ser interpretadas como resultado de rituales iniciáticos. Los/as niños/as son agentes activos dentro de las sociedades paleolíticas, son también autores/as. Respecto a las huellas infantiles dejadas en superficies blandas como la arcilla, muchos investigadores han especulado acerca de su carácter lúdico (BÉGOÜEN Y BREUIL 1958: 101; CLOTTES Y SIMONET, 1972a: 316; PALES, 1974: 5; CLOTTES, 1993: 66,). Incluso algunos han atribuido a los/as nińos/as cierto tipo de representaciones, sobre todo macarronis (SHARPE Y VAN GELDER, 2004; 2006), que podrían responder a actividades psicológico-cognitivas de la infancia como la predilección por determinados lugares o escondrijos en los que hemos podido detectar su presencia (BALBÍN et alii., 2002: 574; CLOTTES Y SIMONET, 1972a: 321). No obstante, creemos precipitado construir una interpretación más sólida en el estado actual de las investigaciones.

\section{CONCLUSIONES}

Los individuos infantiles están presentes en el registro arqueológico de dos formas diferentes. Por un lado tenemos la imagen que la sociedad nos ofrece de ellos a través de su enterramiento y de las representaciones gráficas en las que son protagonistas. Por otro, sus propias manifestaciones (manos y huellas de pies), voluntarias o no, dentro de un territorio o espacio social definido por la comunidad. Estas evidencias materiales apuntan un cambio, a nivel cuantitativo, durante el tecnocomplejo gravetiense, en el que se constata el aumento del número de los enterramientos y del rango de edad de los individuos fallecidos (PREINF: $73 \%$ en el Gravetiense; $27 \%$ posteriormente). A ello se añade el fenómeno gráfico de las manos infantiles «gravetienses», lo que permite discernir cierto protagonismo de los/as niños/as más pequeños/as en esta época. Sin embargo, en etapas más recientes (Magdaleniense, Epigravetiense), los enterramientos infantiles siguen aumentando, aunque levemente, combinándose con representaciones artísticas en vez de con las manos infantiles, lo parece indicar una ligera transformación en la significación y la atención que los grupos humanos paleolíticos prestan a la infancia.

\section{AGRADECIMIENTOS}

Queremos expresar brevemente nuestro sincero agradecimiento a los investigadores, profesores, compañeros y amigos que han reflexionado con nosotros sobre la infancia en el Paleolítico; muy especialmente a M. Bea, P. Utrilla, P. Foucher, C. San Juan-Foucher, J. Ma . Rodanés, A. Ruiz, G. Navarro y G. Fontana. Todos ellos han enriquecido 
de una u otra forma el texto. Sin embargo, todo error u omisión es exclusivamente obra de los autores.

\section{BIBLIOGRAFIA}

ABRAMOVA, Z. A. (1995): L'art paléolithique d'Europe orientale et Sibérie. L'Homme des Origines. Jérôme Million. Grenoble.

AIRVAUX, J. (1998), "Découverte d'une grotte ornée, le Réseau Guy Martin à Lussac-les-Châteaux, Vienne et application d'une méthodologie structurale pour l'étude de l'art préhistorique ", L'Anthropologie, 102 (4). (p. 495-521).

AIRVAUX, J. (2001), L'art préhistorique du Poitou-Charentes. Sculptures et gravures des temps glaciaires. La maison des roches. Paris.

AIRVAUX, J. (2002), "Le Chaffaud, 168 ans après ». Bulletin Préhistoire du SudOuest, 9 (1). (p. 1-52).

ALCALDE DEL RÍO, H., BREUIL, H., SIERRA, L. (1911) Les Cavernes de la Région Cantabrique. Imp. A. Chêne, Monaco.

ARIAS, P; GONZÁLEZ SAINZ, C.; MOURE, A. y ONTAÑÓN, R. (2001, $1^{\text {a }}$ ed. 1999), La Garma. Un descens al Passat. Museu d'Arqueologia de Catalunya. Gener/Abril 2001. Segonda edició corregida.

BAFFIER, D. y GIRARD, M. (1998), Les cavernes d'Arcy-sur-Cure. La maison des roches, Paris.

BALBÍN BEHRMANN, R.; ALCOLEA GONZÁLEZ, J. J. ; GONZÁLEZ PEREDA, M. A. y MOURE ROMANILLO, A. (2002): « Recherches dans le massif d'Ardines: nouvelles galeries ornées de la grotte de Tito Bustillo ». L'Anthropologie, 106. (p. 565-602).

BARRIÈRE, C. (1975), «I. La grotte de Gargas (Hautes-Pyrénées) ». Bulletin de l'Association française pour l'étude du quaternaire, 12 (3-4). (p. 201-203).

BARRIÈRE, C. (1984), "Grotte de Gargas ». V.V.A.A., L'art des Cavernes. Atlas des grottes ornées paléolithiques françaises. Paris. Ministère de la Culture. (p. 514-522).

BÉGOUËN, R.; BREUIL, H. (1958), Les cavernes du Volp. Trois-Frères. Tuc d'Audoubert. Arts et Métiers Graphiques, Paris. 
BELTRÁN, A.; GAILLI, R; ROBERT, R. (1973), La cueva de Niaux. Monografías Arqueológicas, 16. Zaragoza.

BILLY, G. (1919), "L’enfant magdalénien de la grotte du Figuier (Ardèche) ». L'Anthropologie, 83 (2). (p. 223-252).

BINANT, P. (1991), Les sepultures du Paleolithique, Errance, Paris.

BOHIGAS, R.; SARABIA, P. (1988), « Nouvelles découvertes d'art paléolithique dans la Région Cantabrique. La Fuente del Salín. Muñorrodero ». L'Anthropologie. 92. (p. 133-137).

BOIVIN, L.; CLOT, A. y HEIM, J-L. (1986), « Vestiges magdaléniens des déblais de la grotte de Lortet (Hautes-Pyrénées) ». Bulletin de la Société Préhistorique Ariège Pyrénées, XLI. (p. 171-198).

BOROŃ T.; KRÓLIK H. y KOWALSKI, T. (2012), " Les figurines féminines magdaléniennes du site de Wilczyce 10 (district de Sandomierz, Pologne)». CLOTTES J. (Dir.), L'art pleistocìne dans le monde / Pleistocene art of the world / Arte pleistoceno en el mundo. Actes du Congrès IFRAO, Tarasconsur-Ariège, septembre 2010. Symposium "Art mobilier pléistocène ». $\mathrm{N}^{\circ}$ spécial de Préhistoire, Art et Sociétés, Bulletin de la Société Préhistorique Ariège-Pyrénées, LXVLXVI (2010-2011), CD. (p. 1379-1391).

BOSINSKI, G.; D'ERRICO, F. y SCHILLER, P. (2001), Die Gravierten Frauendarstellungen von Gönnersdorf. Franz Steiner. Verlag GMBH. Stuttgart.

BOSINSKI, G. (2011). «Les figurations féminines de la fin des temps glaciaires ». CLUZEL, J.-P. y CLEYET-MERLE, J.J. (Eds.), Mille et une femmes de la fin des temps glaciaires. Paris. Grand Palais. (p. 49-67).

BRŮŽEK, J.; LÁZNIČKOVÁ-GALETOVÁ, M.; GALETA, P. y MAESTRACCI, J. (2012). «Les empreintes de mains dans l'art pariétal : possibilités et limites d'interprétations mises en relief par l'anthropologie médico-légale ». CLOTTES J. (Dir.), L'art pléistocène dans le monde / Pleistocene art of the world / Arte pleistoceno en el mundo. Actes du Congrès IFRAO, Tarasconsur-Ariège, septembre 2010. Symposium "Application des techniques forensiques aux recherches sur l'art pléistocène ». $\mathrm{N}^{\circ}$ spécial de Préhistoire, Art et Sociétés, Bulletin de la Société Préhistorique Ariège-Pyrénées, LXVLXVI (2010-2011), CD. (p. 1197-1206).

CALDWELL, D. (2012), «The identification of the first Paleolithic animal sculpture in the Ile-de-France: the Ségognole 3 bison and its ramifications». 
CLOTTES J. (Dir.), L'art pleistocìne dans le monde / Pleistocene art of the world / Arte pleistoceno en el mundo. Actes du Congrès IFRAO, Tarasconsur-Ariège, septembre 2010. Symposium "Art pléistocène en Europe ». $\mathrm{N}^{\circ}$ spécial de Préhistoire, Art et Sociétés, Bulletin de la Société Préhistorique Ariège-Pyrénées, LXV-LXVI (2010-2011), CD. (p. 415-457).

CANTALEJO, P.; MAURA, R.; ESPEJO Ma DEL M.; RAMOS, J.; MEDIANERO, J.; ARANDA, A. (2006), «Evidencias de frecuentación prehistórica registradas en la cueva de Ardales (Málaga)». SANCHIDRÍAN TORTI, J.L.; MÁRQUEZ ALCÁNTARA, A.M. y FULLOLA I PERICOT, J. M. (Eds.), La Cuenca Mediterránea durante el Paleolitico Superior. 38. $000-10$. 000 años (IV Simposio de Prehistoria Cueva de Nerja) Reunión de la VII Comisión del Paleolítico Superior U.I.S.P.P., (Fundación Cueva de Nerja). (p. 352-365).

CAPITAN, L.; BREUIL, H.; PEYRONY, D. (1924), Les Combarelles aux Eyzies (Dordogne). Masson et Cie Editeurs. Paris.

CERRADA, M. (2007), La mano a través del arte: simbología y gesto de un lenguaje no verbal. Tesis doctoral. Universidad Complutense de Madrid. Facultad de Bellas Artes. Madrid.

CHAPA, T. (2003), « La percepción de la infancia en el mundo ibérico». Trabajos de Prehistoria, 60 (1). (p. 115-138).

CHOllOT, M. (1964). Musée des Antiquités Nationales. Collection Piette: art mobilier préhistorique. Éditions des Musées Nationaux. Paris.

CLOT, A. (1984) : Grotte de Tibiran. En V.V.A.A., Lart des Cavernes. Atlas des grottes ornées paléolithiques françaises. Paris: Ministère de la Culture, p. 536-539.

ClOTTES, J. (1973), "Circonscription de Midi-Pyrénées ». Gallia Préhistoire, 16 (2). (p. 481-523).

CLOTTES, J. (1993), «Ichnologie ». GRAPP. L'Art Pariétal paléolithique techniques et méthodes d'étude. CTHS, Paris. (p. 59-66).

ClOTTES, J. y COURTIN, J. (1994), La grotte de Cosquer. Peintures et gravures de la caverne engloutie. SEUIL. Paris.

CLOTTES, J. y LEWIS-WILLIAMS, D. (1996), Les Chamanes de la Préhistoire. Transe et Magie dans les Grottes ornées, Paris, Le Seuil. 
CLOTTES, J. y LEWIS-WILLIANS, D. (2001), Los chamanes de la Prehistoria. Ariel. Barcelona.

CLOTTES, J. y SIMONNET, R. (1972a), « Le réseau René Clastres de la caverne de Niaux (Ariège)». Bulletin de la Société préhistorique française, 69 (1). (p. 293-323).

CLOTTES, J.; COURTIN, J. y VANRELL, L. (2005), « Nouvelles recherches a la grotte Cosquer (Marseille)». Munibe, 57. (p. 9-22).

CLOTTES, J.; SIMONNET, R. (1972b), «Quelques éléments nouveaux sur le réseau René Clastres de la Caverne de Niaux ». Bulletin de la Société préhistorique de l'Ariège, 27. (p. 21-38).

CRAWFORD, S. (2009), "The Archaeology of Play Things: Theorising a Toy Stage in the 'Biography' of Objets». Childhood in the Past, 2. (p. 56-71).

CRIADO, F. (2012), Arqueológicas. La razón perdida. La construcción de la inteligencia arqueológica. Bellaterra arqueología. Barcelona.

CUNNINGHAM, H. (1996), The History of Childhood. Images of Childhood (C. P. Hwang; M. E. Lamb; I. E. Sigel, eds.), Lawrence Erlbaum Associates Publishers, Mahwah: 27-35.

DAMS, L. (1980), L'art Pariétal De La Grotte Du Roc Saint-cirq. BAR International Séries 79.

DE BLAS CORTINA, M. A. (1997), «En torno a los restos esqueléticos humanos del Paleolítico Superior-Epipaleolítico Cantábrico». Homenaje a Juan Uría Ríu. Universidad de Oviedo. (p. 147-170).

DE MIGUEL, M. P. (2010), «A vision of infancy from osteoarchaeology: from Prehistory to the Middles Ages ». Complutum, 21(2). (p. 135-154).

DELLUC, B. y DELLUC, G. (2009), «Art paléolithique en Périgord. Les représentations humaines pariétales ". L'Anthropologie, 113. (p. 629-661).

DROUOT, E., (1984). "Grotte Bayol ». V.V.A.A., L'art des Cavernes. Atlas des grottes ornées paléolithiques françaises. Paris. Ministère de la Culture. (p. 323-326).

DUDAY, H. y GARCIA M-A. (1983), «Les empreintes de l’homme préhistorique. La grotte du Pech Merle à Cabrerets (Lot), une relecture significative des traces de pieds humains ». Bulletin de la Société préhistorique française, 80 (7). (p. 208-215). 
DUHARD, J-P. (1992), « Les figurations humaines sculptées et gravées du Mas d'Azil (Ariège) ». Gallia Préhistoire, 34. (p. 289-301).

DUHARD, J-P. (1993), Réalisme de l'image féminine paléolithique. Editions du C.N.R.S. Cahiers du Quaternaire no ${ }^{19}$. Paris.

DUHARD, J-P. (1996), Réalisme de l'image masculine paléolithique. Éditions Jérôme Million. Grenoble.

EIGENWÖGERER, Th.; FRIESINGER, H.; HANDEL, M.; NEUGENBLAUER, Ch.; SIMON, U. y TESCHLER-NICOLA, M. (2006), „Upper palaeolithic infant burials«. Nature, 444. (p. 285).

FERNÁNDEZ ALGABA, M.; ADÁN, G. Y ARSUAGA, J.L. (2009), «Grafismo rupestre paleolítico del valle del Trubia (Santo Adriano, Asturias): Los sitios de Cueva Pequeña y el Camarón de las Ciervas de Los Torneiros». Arte rupestre das Transiçoes. Congresso Internacional da IFRAO 2009. Piauí, Brasil. (p. 1211-1223).

FOUCHER, P., SAN JUAN-FOUCHER, C. y RUMEAU, Y. (2007), La Grotte de Gargas. Un siécle de découvertes. Édition Communauté de communes du canton de Saint- Laurent-de-Neste.

GARCÍA, M-A (2005), "Ichnologie générale de la grotte Chauvet ». Bulletin de la Société préhistorique française, 102 (1). (p. 103-108).

GARCIA, M-A. (1999), «La piste de pas humains de la grotte Chauvet à Vallonpont-d'Arc ». INORA, 24. (p. 1-4).

GARCÍA, M-A.; DUDAY, H. y COURTAUD, P. (1990), "Les empreintes du Réseau Clastres ». Bulletin de la Société Préhistorique Ariège, XLV. (p. 167-174).

GIBAJA, J.F.; MAJÓ, T.; CHAMBON, P.; RUIZ, J. y SUBIRÀ, M. E. (2010), «Prácticas funerarias durante el neolítico. Los enterramientos infantiles en el noreste de la Península Ibérica». Complutum, 21 (2). (p. 47-68).

GOLOMSHTOK E.A. (1933), "Trois gisements du Paléolithique supérieur russe et sibérien ». L'Anthropologie, 43. (p. 333-346).

GONZÁLEZ MORALES, M. R. (2007), «La imagen del sexo en el Paleolítico». CELESTINO PÉREZ, S. (Ed.), La imagen del sexo en la Antigüedad. TusQuets Editores. Barcelona. (p. 49-66).

GRAZIOSI, P. (1962), Levanzo. Pitture e incisioni. Firenze. Sansoni. 
GROENEN, M. (2000), Sombra y luz en el arte paleolitico, Ariel. Barcelona.

GUY, E. (2003), «Os d'oiseau dit la «Scène d'initiation». CLOTTES, J. y DELPORTE, H. (Eds.), La grotte de La Vache (Ariège). Fouilles Romain Robert. II. L'art mobilier. Musée des Antiquites Nationales. Éditions de la Réunion des Musées Nationaux. Paris. (p. 297).

HEIM, J-L. (1991), "L'enfant magdalénien de la Madeleine ». L'Anthropologie, $95(2 / 3):$ 611-638.

HENRY-GAMBIER, D. (2002), " Les fossiles de Cro-Magnon (Les-Eyzies-deTayac, Dordogne) : nouvelles données sur leur position chronologique et leur attribution culturelle ». Páleo, 14. (p. 201-204).

HENRY-GAMBIER, D. (2005), «Évolution des pratiques funéraires en Italie au Paléolithique supérieur ». VIALOU, D.; RENAULT-MISKOVSKY, J.; y PATOU-MATHIS, M. (dirs.), Comportements des hommes du Paléolithique moyen et supérieur en Europe: territoires et milieux. Actes du Colloque du G.D.R. 1945 du CNRS, Paris. 8-10 janvier 2003. Liège. ERAUL 111. (p. 213-229).

HENRY-GAMBIER, D. (2008), "Comportement des populations d'Europe au Gravettien : pratiques funéraires et interprétations ». Páleo, 20. (p. 399-438).

HERNÁNDEZ, M. S. y SOLER, J. (Eds.), Arte Rupestre en la España Mediterránea. MARQ. Alicante. (p. 341-377).

HOCSMAN, S. (2006), «Producción de bifaces y aprendices en el sitio Quebrada Seca 3-Antofagsta de la Sierra, Catamarca-(5500-4500 años A.P.)». NIELSEN, A.; RIVOLTA, M.C.; SELDES, V.; VÁZQUEZ, M.M. y MERCOLLI, P. H. (Comp.), Producción y circulación prehispánicas de bienes en el sur andino. Editorial Brujas Córdoba. Argentina. (p. 55-82).

HOEBEL, E. A y WEAVER, T. (1984), Antropología y experiencia humana. Omega. Barcelona.

IAKOVLEVA, L. (2008), " La décoration corporelle, une manifestation du système socio-symbolique du Paléolithique supérieur ancien en Europe orientale (d'après les sépultures de Kostienki 15 et de Soungir) ». Bulletin de la Société Préhistorique Ariège-Pyrénées, LXIII. (p. 61-71).

IRISH, J. D.; BRATLUND, B.; SCHILD, R.; KOLSTRUP, E.; KRÓLIK, H.; MAŃKA, D. y BOROŃ T. (2008), «A late Magdalenian perinatal human 
skeleton from Wilczyce, Poland». Journal of Human Evolution, 55 (4). (p. 736-740).

KAMP, K. (2001a), «Where have all the children gone? The Archaeology of childhood». Journal of Archaeological Method and Theory, 8 (1). (p. 1-34).

KAMP, K. (2001b), «Prehistoric Children Working and Playing: a Southwestern Case Study in Learning Ceramics». Journal of Anthropological Research, 57 (4). (p. 427-450).

KAMP, K. (2010), «Entre el trabajo y el juego: perspectivas sobre la infancia en el suroeste norteamericano». Complutum, 21 (2). (p. 103-120).

LACALLE RODRÍGUEZ, R (1996), «El símbolo de la mano en arte paleolítico». Zephyrus, 49. (p. 273-279).

LEROI-GOURHAN, A. (1967), "Les mains de Gargas, essai pour une étude d'ensemble ». Bulletin de la Société Préhistorique Française, LXIV. (p. 107-122).

LEROI-GOURHAN, A. (1971), Préhistoire de l'Art Occidental. Éditions d'Art Lucien Mazenod. Paris.

LILLEHAMMER, G. (1989), "A child is born. The child's world in an archaeological perspective». Norwegian Archaeological Review, 22 (2). (p. 89-105).

LILLEHAMMER, G. (2010), «Archeology of Children». Complutum, 21(2). (p. 14-45).

LORBLANCHET. M. (1995), Les grottes ornées de la Préhistoire. Nouveaux regards. Paris.

LORBLANCHET, M. (2010), Art pariétal. Grottes ornées du Quercy. Editions du Rouergue.

MAUREILLE, B. (2004), Les premières sépultures. Le Pommier. Paris.

MAY, F. (1986), Les sépultures préhistoriques. Étude Critique. Editions du CNRS. Paris.

MÉLARD, N. (2008), « Pierres gravées de La Marche à Lussac-les-Château (Vienne). Techniques, technologie et interprétations ». Gallia Préhistoire, 50. (p.143-268).

MOLINERO ARROYABE, J. T. (2002), «La Lastrilla». VV.AA, Las cuevas con arte paleolitico en Cantabria. ACDPS. Santander. (p. 309-312). 
MOURE-ROMANILLO, J-A; GONZÁLEZ MORALES, M.R.; GONZÁLEZ SAINZ, C. (1984-1985), «Las pinturas paleolíticas de la cueva de la Fuente del Salín (Muñorrodero, Cantabria)». Ars Praehistorica, III-IV. (p. 13-23).

MÜLLER-KARPE, G. (1966), Handbuch der Vorgeschichte, Band I. C.H.Beck'sche Verlagsbuchhandlung. München.

NÁJERA, T.; MOLINA, F.; JIMÉNEZ-BROBEIL, S.; SÁNCHEZ ROMERO, M.; AL OUMAOUI, I.; ARANDA, G.; DELGADO-HUERTAS, A. y LAFFRANCHI, Z. (2010), «La población infantil de la Motilla del Azuer: un estudio bioarqueológico». Complutum, 21 (2). (p. 69-102).

OLÀRIA, C. (2008), „Restos y tumbas infantiles y juveniles en la Prehistoria europea: del Musteriense al Mesolítico«. GUSI JENER, J,; MURIEL, S. y OLÀRIA, C. (Coords.), Nasciturus. Infans, puerulus. Vobis mater terra. La muerte en la infancia. Diputació de Castelló, Servei d'investigacions Arqueològiques i Prehistòriques.

PALES, L (1976), Les empreintes de pieds humains dans les cavernes, Archives de l'Institut de Paléontologie humaine, Mémoire 36, Masson, Paris.

PALES, L; TASSIN DE SAINT PÉREUSE, M. (1976), Les gravures de La Marche. II. Les Humains. (Éditions Ophrys), Paris.

PALMA DI CESNOLA, A. (1993), Il paleolitico superiore in Italia. Introduzione allo studio. Garlatti e Razzai Editori. Firenze.

PIETTE, E. (1895), «La station de Brassempouy et les statuettes humaines de la période glyptique ». L'Anthropologie, 6. (p. 129-151).

PIETTE, E. (1907), L'art pendant l'Âge du Renne. Albun de cents planches dessinées par J. Pilloy (Masson et Cie). Paris.

PIGEOT, N. (1987), Magdaléniens d'Étiolles. Économie de débitage et organisation sociales (l'unité d'habitation U5). XXV Supplément à GALLIA PRÉHISTOIRE, éditions du CNRS, Paris.

POLITIS, G. (1998), «Arqueología de la infancia. Una perspectiva etnográfica». Trabajos de Prehistoria, 55 (2). (p. 5-19)

POLITIS, G. (1999), «La actividad infantil en la producción del registro arqueológico de cazadores-recolectores». Revista do Museu de Arqueología e Etnologia, 3. (p. 263-283). 
RIPOLL LÓPEZ, S; RIPOLL PERELLÓ, E; COLLADO GIRALDO, H. (1999), Maltravieso. El santuario extremeño de las manos. Memorias 1. Junta de Extremadura. Consejería de Cultura. Mérida.

RUIZ REDONDO, A. (2010), «Una nueva revisión del Panel de las Manos de la cueva de El Castillo (Puente Viesgo, Cantabria)». Munibe, 61. (p. 17-27).

RUIZ VENTURA, J; GARCÍA SIVOLI, C; MARTÍNEZ-MORENO, J; SUBIRÀ DE GALDÁCANO. (2006), «Los restos humanos del Tardiglaciar de Balma Guilanyà» SANCHIDRÍAN TORTI, J.L.; MÁRQUEZ ALCÁNTARA, A.M. y FULLOLA I PERICOT, J. M. (Eds.), La Cuenca Mediterránea durante el Paleolítico Superior. 38. 000 - 10. 000 años (IV Simposio de Prehistoria Cueva de Nerja) Reunión de la VII Comisión del Paleolítico Superior U.I.S.P.P. (Fundación Cueva de Nerja). (p. 458-466).

SACCASYN DELLA SANTA, E. (1947), Les figures humaines du Paléolithique supérieur euroasiatique. De Sikkel. Anvers.

SACCHI, M. (2010), "Algunos apuntes sobre la Arqueología de la Infancia: exploración de vías metodológicas para su definición». Revista de Antropología Experimental, 10. (p. 281-292).

SAHLY, A. (1966), Les mains mutilées dans l'art préhistorique. Maison Tunisienne de l'édition, Túnez.

SÁNCHEZ ROMERO, M (2010), «iEso no se toca! Infancia y cultura material en arqueología. Complutum, 21 (2). (p. 9-13).

SÁNCHEZ ROMERO, M. (2007), «Actividades de mantenimiento en la Edad del Bronce del sur peninsular: El cuidado y la sociabilización de individuos infantiles». Complutum, 18. (p. 25-34).

SANCHIDRÍAN, J.M. (2001), Manual de Arte Prehistórico. Ariel Prehistoria. Barcelona.

SHARPE, K. y VAN GELDER, L. (2004), "Children and Paleolithic 'art': indications from Rouffignac Cave, France». International Newsletter on Rock Art (INORA), 38. (p. 9-17).

SHARPE, K. y VAN GELDER, L. (2006), «Evidence of cave marking by Paleolithic children». Antiquity, 80 (310). (p. 937-947). 
TIXIER, J.; MARMIER, F. y TRÉCOLE, G. (1976), Le campement préhistorique de Bordj Mellala. Ouargla, Algérie, Crecle de recherches et d'études préhistoriques, Paris.

TOSELLO, G, FRITZ, C (2005), « La Vénus et le Sorcier Les figurations humaines pariétales au Magdalénien ". Bulletin de la Société Préhistorique Ariège-Pyrénées, 60. (p. 7-24).

TYMULA, S. (2002), L'art solutréen du Roc de Sers (Charente). Éditions de la Maison des Sciences de l'Homme. Paris.

UTRILlA, P. (2005), «El Arte Rupestre en Aragón. 100 Años después de Calapatá». HERNÁNDEZ, M. S. y SOLER, J. (Eds.). Arte Rupestre en la España Mediterránea. MARQ. Alicante. (p. 341-377).

UTRILLA, P; BALDELLOU, V; BEA, M y VIÑAS, R. (e.p.), «La cueva de la Fuente del Trucho (Asque-Colungo, Huesca). Una cueva mayor del arte gravetiense». Actas del Coloquio Internacional Gravetiense Cantábrico, estado de la cuestión. Museo Nacional y Centro de Investigación de Altamira (Santillana del Mar). 20-22 de octubre de 2011.

UTRILlA, P; MONTES, L. (2007), «El Paleolítico superior al sur de los Pirineos. Contactos entre fronteras». CAZALS, N.; GONZÁLEZ URQUIJO, J. y TERRADAS, X. (Eds), Frontières naturelles et frontières culturelles dans les Pyrénées préhistoriques (Actas de la Table Ronde Tarascon/sur/Arige, Mars 2004) Monografías del IIIPC. (PubliCan Ediciones, Santander). (p. 205-223).

VALLE GÓMEZ, A; SERNA GANCEDO, M. L. (2002), «Fuente del Salín». V.AA Las cuevas con arte paleolitico en Cantabria. ACDPS. Santander. (p. 83-88).

VALLOIS, H-V. (1971), "Le crâne trépané magdalénien de Rochereil ». Bulletin de la Société Préhistorique Française, 68 (2). (p. 485-495).

VANHAEREN, M. y D’ERRICO, F. (2001), «La aderezo de l'enfant de la Madeleine (fouilles Peyrony). Un nouveau regard sur l'enfance au Paléolithique supérieur ». Páleo, 13. (p. 201-240).

VIALOU, D. (1986), L'art des grottes en Ariège magdalénien. XXIIe supplément à GALLIA PREHISTOIRE. Éditions du C.N.R.S. Paris. 
VILÀ, A. (2006), «Propuesta de evaluación de la metodología arqueológica». Etnoarqueología de la Prehistoria. Más allá de la Analogía. Madrid. CSIC. Serie de Treballs d'Etnoarqueologia, 6. (p. 61-76).

VLCEK, E (1991), «L'Homme fossile en Europe Centrale». L'Anthropologie, 95 (2/3). (p. 409-472).

VVAA. (2006), La infancia en el arte. Unicef. Madrid.

WELTÉ, A-C. y LAMBERT, G-N. (2005), «Aux origines de la Spiritualité : la notion de transcendance au Paléolithique ". L'Anthropologie, 109. (p. 723-741). 